\title{
The cost of providing mechanical thrombectomy in the UK NHS: a micro-costing study
}

\author{
Authors: Joyce S Balami, ${ }^{\mathrm{A}}$ Diamuid Coughlan, ${ }^{\mathrm{B}}$ Phil M White, ${ }^{\mathrm{C}}$ Peter McMeekin, ${ }^{\mathrm{D}}$ Darren Flynn, ${ }^{\mathrm{E}}$ \\ Christine Roffe, ${ }^{\mathrm{F}}$ Indira Natarajan, ${ }^{\mathrm{G}}$ Jayan Chembala, ${ }^{\mathrm{G}}$ Sanjeev Nayak, ${ }^{\mathrm{H}}$ Ivan Wiggam, ${ }^{\mathrm{I}}$ Peter Flynn, ${ }^{\mathrm{J}}$ \\ Robert Simister, ${ }^{\mathrm{K}}$ Yazen Sammaraiee, ${ }^{\mathrm{L}}$ Don Sims, ${ }^{\mathrm{M}}$ Kurdow Nader, ${ }^{\mathrm{N}}$ Anand Dixit, ${ }^{\mathrm{O}}$ Dawn Craig, ${ }^{\mathrm{P}}$ Hannah Lumley, ${ }^{\mathrm{Q}}$ \\ Stephen Rice, ${ }^{\mathrm{R}}$ David Burgess, ${ }^{\mathrm{S}}$ Lisa Foddy, ${ }^{\mathrm{T}}$ Emer Hopkins, ${ }^{\mathrm{U}}$ Beverley Hudson, ${ }^{\mathrm{V}}$ Rachael Jones, ${ }^{\mathrm{W}}$ Martin A James, ${ }^{\mathrm{X}}$ \\ Alastair M Buchan, ${ }^{\mathrm{Y}}$ Gary A Ford ${ }^{\mathrm{Z}}$ and Alastair M Gray ${ }^{\mathrm{A}}$
}

\section{Introduction}

The clinical efficacy and cost-effectiveness of mechanical thrombectomy (MT) for the treatment of large vessel occlusion stroke is well established, but uncertainty remains around the true cost of delivering this treatment within the NHS. The aim of this study was to establish the cost of providing MT within the hyperacute phase of care and to explore differences in resources used and costs across different neuroscience centres in the UK.

Authors: Aresearch fellow and consultant physician, Centre for Evidence-Based Medicine, Oxford, UK and Norfolk and Norwich University Hospitals NHS Foundation Trust, Norwich, UK; ${ }^{B}$ research associate, Newcastle University, Newcastle upon Tyne, UK; ${ }^{\text {C }}$ professor of neuroradiology and honorary consultant neuroradiologist, Newcastle University, Newcastle upon Tyne, UK and Newcastle upon Tyne Hospitals NHS Foundation

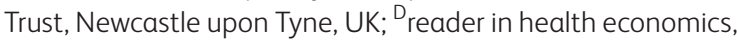
Northumbria University, Newcastle upon Tyne, UK; E $_{\text {reader in }}$ long-term health conditions, Teesside University, Middlesbrough, UK; ${ }^{\mathrm{F}}$ consultant stroke physician and professor of stroke medicine, University Hospitals of North Midlands NHS Foundation Trust, Stoke-on-Trent, UK and Keele University, Keele, UK; ${ }^{G}$ consultant stroke physician, University Hospitals of North Midlands NHS Foundation Trust, Stoke-on-Trent, UK and Keele University, Keele, UK; ${ }^{H}$ consultant neuroradiologist, University Hospitals of North Midlands NHS Foundation Trust, Stoke-on-Trent, UK and

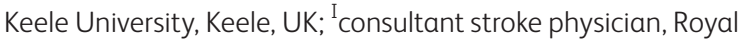
Victoria Hospital, Belfast, UK; ' consultant neuroradiologist, Royal Victoria Hospital, Belfast, UK; ${ }^{\text {K}}$ consultant neurologist,

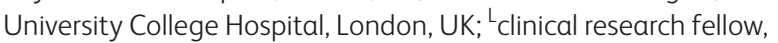
University College Hospital, London, UK; ${ }^{M}$ consultant stroke physician, University Hospitals Birmingham NHS Foundation Trust, Birmingham, UK; ${ }^{N}$ consultant neuroradiologist, University Hospitals Birmingham NHS Foundation Trust, Birmingham, UK; ${ }^{0}$ consultant stroke physician, Newcastle upon Tyne Hospitals NHS Foundation Trust, Newcastle upon Tyne, UK; P professor (evidence synthesis), Newcastle University, Newcastle upon Tyne, UK; ${ }^{\text {research }}$ assistant, Newcastle University, Newcastle upon Tyne, UK; ${ }^{R}$ senior research associate, Newcastle University, Newcastle upon Tyne, UK;

\section{Method}

This was a multicentre retrospective study using microcosting methods to enable a precise assessment of the costs of MT from an NHS perspective. Data on resources used and their costs were collected from five UK neuroscience centres between 2015 and 2018.

Results

Data were collected on 310 patients with acute ischaemic stroke treated with MT. The mean total cost of providing MT and inpatient care within 24 hours was $£ 10,846$ (95\% confidence interval (CI) 10,527-11,165) per patient. The main driver of cost was MT procedure costs, accounting for $73 \%$ ( $£ 7,943 ; 95 \%$ CI $7,649-8,237$ ) of the total 24 -hour cost. Costs were higher for patients treated under general anaesthesia ( $£ 11,048$; standard deviation (SD) 2,654) than for local anaesthesia ( $£ 9,978 ;$ SD 2,654), mean difference $£ 1,070$ (95\% CI 381-1,759; $p=0.003)$; admission to an intensive care unit (ICU; $£ 12,212$; SD 3,028) against for admission elsewhere ( $£ 10,179 ;$ SD 2,415$)$, mean difference $£ 2,032$ (95\% CI 1,345-2,719; $\mathrm{p}<0001$ ).

\footnotetext{
Slay member, North East and North Cumbria Stroke Patient \& Carer Panel, Newcastle upon Tyne, UK; ' 'senior operational services manager, University Hospitals of North Midlands NHS Foundation Trust, Stoke-on-Trent, UK and Keele University, Keele, UK; ${ }^{U}$ coordinator regional stroke network, Health and Social Care Board,

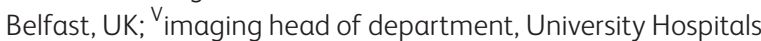
Birmingham NHS Foundation Trust, Birmingham, UK; ${ }^{W_{\text {senior }}}$ advanced nurse practitioner, University Hospitals Birmingham NHS Foundation Trust, Birmingham, UK; ${ }^{X}$ consultant stroke physician, Royal Devon and Exeter NHS Foundation Trust, Exeter, UK; Y professor of stroke medicine and honorary consultant neurologist, University of Oxford, Oxford, UK and John Radcliffe Hospital, Oxford, UK; Z professor of stroke medicine, Oxford University, Oxford, UK, visiting professor, Newcastle University, Newcastle upon Tyne, UK and consultant stroke physician, John Radcliffe Hospital, Oxford, UK; ${ }^{\text {AA }}$ professor of health economics, University of Oxford, Oxford, UK
} 
The mean cost within 72 hours was $£ 12,440$ (95\% CI 10,628-14,252). The total costs for the duration of inpatient care before discharge from a thrombectomy centre was $£ 14,362$ (95\% CI 13,603-15,122).

Conclusions

Major factors contributing to costs of MT for stroke include consumables and staff for intervention, use of general anaesthesia and ICU admissions. These findings can inform the reimbursement, provision and strategic planning of stroke services and aid future economic evaluations.

KEYWORDS: Acute ischaemic stroke, treatment, mechanical thrombectomy, costs, micro-costing

DOI: 10.7861/clinmed.2019-0413

\section{Introduction}

The total healthcare and societal costs of stroke to the NHS in England, Wales and Northern Ireland are estimated at $£ 1.74$ billion at 1 year and $£ 3.6$ billion at 5 years after admission (mean cost per patient $=£ 46,039)$. ${ }^{1}$ The clinical and cost effectiveness of mechanical thrombectomy (MT) with or without intravenous thrombolysis (IVT) for the treatment of large vessel occlusion stroke are both well established. ${ }^{2-6}$ Despite the evidence, detailed costs of delivering MT within most healthcare economies, including the UK NHS, remain unclear. Evidence on the main drivers of cost and its variability are also sparse.

Given the limited availability of thrombectomy centres and the ongoing restructuring of services, knowledge of the detailed costs of MT is essential for efficient resource allocation. In England, the current NHS tariff for providing MT is $£ 11,750$, but it is not clear how accurately this reflects routine clinical care or whether the tariff only covers the intervention, the hyperacute phase or the entire care pathway at the MT centre. ${ }^{7}$ The National Institute for Health and Care Excellence (NICE) has previously published prices for thrombectomy devices of two types, with ranges from $£ 1,900$ to $£ 5,000$ for stent-retrievers and from $£ 550$ to $£ 1,349$ for thrombo-aspirators (per unit and excluding VAT). ${ }^{8}$ From the Sentinel Stroke National Audit Programme (SSNAP) data, in 2018-19, of all the 87,635 acute ischaemic stroke (AIS) patients, 1,200 ( 1.45\%) were treated with MT in England, Wales and Northern Ireland. ${ }^{9}$ The number treated with MT is expected to rise as thrombectomy services evolve, particularly considering the ongoing plans for reorganisation and expansion of thrombectomy services.

Better evidence on the resources required and the real costs of providing MT in routine clinical settings would be invaluable not only to healthcare managers and commissioners, clinicians and researchers, but also to policy makers and planners in many European health systems. The need for more reliable and up-todate evidence is particularly acute in the UK, where the challenge is to improve access for the 10,000 UK stroke patients annually who could potentially benefit from $\mathrm{MT}^{10}$

This study, therefore, aims to establish the cost of delivering MT in the clinical setting within the UK NHS (particularly within the hyperacute phase of care), to explore the resources used in care provision and to explore cost variations between the different models of care and across five treatment centres in the UK.

\section{Methods}

\section{Study design}

A multicentre retrospective cost analysis of stroke patients treated with MT with or without IVT was performed. A micro-costing approach was adopted from the perspective of the UK NHS, aiming to undertake the 'direct enumeration and costing-out of every input consumed in the treatment of a particular patient'.11,12 Such micro-costing data at the individual level can provide more precise estimates of costs and give insight into heterogeneity. The study was guided by economic principles and involved collection of detailed resource use information, notably the quantity and unit costs of all resources used along each individual patient's treatment pathway (supplementary material S1). Data were collected on patients treated in line with routine clinical practice in five experienced and active thrombectomy centres.

\section{Resource use data collection}

The resources on which detailed information were collected included pre-hospital transportation; arrival at hospital, either to the emergency department, the radiology department or the stroke unit; imaging; procedural and staffing costs of thrombectomy; hospital or intensive care unit (ICU) stay after interventions; personnel hours; interventional radiology suite (IRS) or theatre usage; and type of anaesthesia used. Hospital stay up to 24 hours (ICU or stroke unit), inpatient care within 72 hours and overall duration of stay at the MT centre costs were determined.

\section{Cost estimation}

An NHS costing perspective was adopted and the cost per patient was measured and valued in pounds sterling at 2017-2018 prices. ${ }^{13}$ Where unit costs were only available for previous years, a gross domestic product deflator was used to adjust. ${ }^{14}$ The average cost per hour of staff time in different categories was obtained from the Personal Social Services Research Unit (PSSRU). ${ }^{15}$ The cost of the individual thrombectomy device was obtained from each of the participating hospitals' finance departments, as the national prices quoted by NICE have only been recently agreed. Other unit costs were collected from NHS healthcare resource group reference costs, NHS England specialised commissioning teams, individual participating hospitals' finance departments, expert opinions and published data. The cost of alteplase for thrombolysis was taken from the British National Formulary. ${ }^{16}$ The results are presented in pounds sterling and converted to euros using 2018 World Bank purchasing power parity in supplementary material. ${ }^{17}$

\section{Study sites}

Five regional neuroscience centres in the UK participated in the micro-costing study. The centres were University Hospitals of North Midlands NHS Trust; Royal Victoria Hospital, Belfast; The Newcastle upon Tyne Hospitals NHS Foundation Trust; Queen Elizabeth Hospital Birmingham and University College London Hospitals NHS Foundation Trust. These are all neuroscience centres which provide MT both to local patients transferred directly (known as 'mothership') and to those initially transported to the nearest IVT centre and subsequently moved by secondary transfer to the neuroscience centre for MT (known as 'drip-and-ship'). Additionally, in some regions, patients may be directly transferred 
to MT centres bypassing nearest IVT centre, before repatriation to their primary hospital (described as 'direct transfer' or 'bypass').

The direct transfer patients differ from the mothership patients because they are repatriated back to their local hospitals. Similarly, the direct transfer patients differ from the drip-and-ship patients, as they are taken first to MT centre for imaging, thrombolysis and thrombectomy before repatriation.

All patients included in the study across the five centres had moderate to severe ischaemic strokes (National Institutes of Health Stroke Scale (NIHSS) $\geq 6$ for anterior circulation stroke and NIHSS $<6$ for basilar thrombus) treated with MT with or without IVT. Patients were treated in both mothership and drip-andship models of care. Given the absence of direct patient contact and the retrospective collation and analysis of anonymised routinely collected data, no research ethics approval was required. Nonetheless, approval for participation was obtained from each hospital's research and development department.

\section{Statistical analysis}

Data on resource quantities and unit costs of the different cost components were collected on a bespoke MS Excel spreadsheet. The populated spreadsheet was then exported to IBM SPSS version 25 to undertake descriptive and inferential statistical analyses.

The unit costs were presented as mean, standard deviation (SD) and $95 \%$ confidence interval (CI), median (range) for continuous variables, and with percentages for categorical variables. Mean cost differences were compared using Student's t-tests. A p value of $<0.05$ was considered statistically significant.

\section{Results}

\section{Patient characteristics and service configuration}

Individual patient data were collected on 310 patients with AIS treated with MT with or without IVT from the five neuroscience centres treated between January 2015 and December 2018. One-hundred and ninety-two patients were treated in a mothership model and 118 in drip-and-ship. Among the mothership group, a small proportion of patients ( $n=26)$ were 'bypass' - directly transferred to MT centre before repatriation to primary hospital.

The median age of patients was 72 years (range 23-101); $56(18 \%)$ patients were $>80$ years old and $161(52 \%)$ were male. The median baseline NIHSS score on admission was 17 (range 3-35) and median 24-hour NIHSS was 9 (range 0-35). The median length of inpatient stay at the MT centre was 6 days (range 1-103) and for repatriated patients it was 4 days (range 1-72).

\section{Costs}

The mean total cost of providing MT and subsequent inpatient care over the first 24 hours was $£ 10,846$ (95\% CI 10,527-11,165) per patient. The main cost driver was the thrombectomy procedure, which accounted for $73 \%(£ 7,943 ; 95 \%$ CI $7,649-8,237)$ of the cost. The various components of costs are summarised in supplementary material S2, Table S1.

Costs were significantly higher for patients admitted to ICU straight after the procedure $(£ 12,212 ;$ SD 3,028) than for those not admitted to ICU ( $£ 10,179 ;$ SD 2,415), mean difference $£ 2,032$ (95\% CI 1,345-2,719), $p<0001$. Similarly, patients with carotid stenosis who required carotid stenting and/or angioplasty during the thrombectomy procedure had higher costs ( $£ 12,474 ;$ SD 2,280) than those without additional stenting/angioplasty ( $£ 10,705 ;$ SD 2,801), mean difference $£ 1,768$ (95\% CI 795-2,741), $p=0.001$ (supplementary material S2, Table S2).

The mean total cost of providing MT and subsequent inpatient care up to 72 hours of care was $£ 12,440$ (95\% CI 10,628-14,252). The total cost up to final discharge to all different destinations (home, community hospital, care home etc) from the MT centre was $£ 14,362$ (95\% CI 13,603-15,122). The main driver of cost post-MT provision was ICU stay greater than 24 hours ( $£ 20,033$; SD 11,130$)$ compared with patients not admitted to ICU ( $£ 11,823$; SD 3,510), mean difference $£ 8,210$ (95\% CI 4,833-11,588); $\mathrm{p}<0001$ (supplementary material S2, Table S2).

\section{Subgroups}

\section{Different regions and centres}

The 24-hour costs varied widely between centres, ranging from $£ 8,858$ (SD 1,727) to $£ 12,942$ (SD 3,246) with a significant mean difference of $£ 4,084$ ( $95 \%$ CI 5,087-3,080), $p<0.001$. The cost to discharge from the MT centre also varied widely, from $£ 11,081$ (SD 4,245) to $£ 18,889$ (SD 5,047) with mean difference of $£ 7,808$ (95\% CI 9,583-6,033), $p<0.001$. The cost components per centre are summarised in supplementary material S2, Table S3.

\section{Mothership vs drip-and-ship}

For the 192 mothership patients, mean 24-hour costs of MT were $£ 10,780$ (SD 2,786) compared with $£ 10,989$ (SD 2,832) for the 118 drip-and-ship patients, mean difference $£ 209$ (95\% CI 453-870), $p=0.54$ in favour of mothership. There were statistically significant differences between mothership and drip-and-ship patients in transportation costs ( $£ 333$ (SD 234) vs $£ 568$ (SD 258); mean difference $£ 236$ (95\% Cl 178-293); $p<0.001)$; in emergency hospital attendance costs ( $£ 532$ (SD 94) vs $£ 616$ (SD 148); mean difference $£ 85$ (95\% Cl 54-115); $p<0.001$ ); and imaging costs ( $₹ 337$ (SD 97) vs $£ 485$ (SD 223); mean difference $£ 148$ (95\% $\mathrm{Cl} 104-192) ; p<0.001)$. The number of patients treated in the mothership vs drip-and-ship for each of the five centres are in supplementary material \$2, Table \$4.

\section{Direct transfer or 'bypass' vs drip-and-ship}

Among the 310 patients, only 26 were transferred directly to a thrombectomy centre before repatriation to primary hospital. The mean 24 -hour cost of MT was $£ 11,858$ (SD 4,379) for direct transfer and $£ 10,989$ (SD 2,832) for drip-and-ship, mean difference $£ 869$ (95\% CI 744-2743), $p=0.36$. The mean total cost until discharge from MT centre was $£ 16,499$ (SD 7,612) for direct transfer and $£ 13,066$ (SD 5,262) for drip-and-ship, mean difference $£ 3,432$ (95\% Cl 90-6,775), $p=0.45$ in favour of dripand-ship. There was a statistically significant difference between direct transfer compared with drip-and-ship in imaging cost ( $£ 334$ (SD 67) vs $£ 485$ (SD 223); mean difference $£ 151$ (95\% $\mathrm{Cl} 102-200) ; p<0.001)$. No significant differences were found in costs of transportation, emergency hospital attendance (EHA) or procedure cost (supplementary material S2, Table S5).

\section{Discussion}

To our knowledge, this is the first multicentre comprehensive micro-costing study of MT treatment for AIS in Europe and the only study that captures the whole treatment pathway from 
prehospital transportation through to hospital arrival, treatment and discharge from the MT centre. It is undertaken from the UK NHS perspective and provides a detailed insight into the true costs of providing MT in routine clinical care across five different centres. Our findings describe the cost of providing MT in a range of realworld settings in a major western European healthcare economy.

Our estimated mean cost per patient of $£ 10,846$ (SD 2,796) within 24 hours post MT falls within the current NHS England tariff (reimbursement) of $£ 11,750$ (excluding market forces factor (MFF)) and the reimbursement of $£ 12,000$ for Northern Ireland, omitting any allowance for units and excluding potential additional costs related to ICU admission or carotid stenting. ${ }^{18}$ However, the cost of care for the first 72 hours post-MT, especially for patients on ICU for more than 24 hours, is higher than the current NHS England tariff. The final reimbursement amount for each neuroscience centre varies depending on the MFF index for each region, which ranges from 1.025707 to $1.276880 .{ }^{19}$ It is also dependent on completion of the SSNAP database and activity being reported via Secondary Uses Service; however, it is unclear whether the reimbursement is only for the intervention or for the whole duration of stay at the MT centre.

Thrombectomy procedure costs accounted for three-quarters of the overall total thrombectomy cost in our study, particularly the cost within 24 hours. The main constituents of costs were thrombectomy devices; staffing; use of general anaesthesia with subsequent admission to ICU / high-dependency unit (HDU); and postoperative care. Patients treated with carotid stenting during the procedure also incurred higher costs.

A previous European economic analysis which used a microcosting approach but only included procedural costs, reported that the cost of the stent retriever (SR) and other consumables was $£ 8,365 .{ }^{20}$ This was higher than the total of $£ 7,943$ (SD 2,631) identified by the current study. The previous (2015) study was also based on just one centre in the UK, while the current study covers multiple thrombectomy devices and involves five centres across different regions of the UK. In another single centre study by the same group in 2016, the procedural cost of MT was reported to be $£ 7,283$ including the costs of devices/consumables and staffing. ${ }^{21}$ This was within the range of the current study but, again, only SR thrombectomy devices were costed.

A Swedish economic analysis was based on just 10 patients treated with MT from a single centre using predominately SR. ${ }^{22}$ It reported MT costs comprising staff (neuro-interventionalist and neurologist), anaesthesia, surgery time, neurointensive care and MT devices (mainly SR) of $£ 9,352$. This was higher than the average $£ 7,943$ from the current study; however, the small sample size provided little insight into variability or heterogeneity or sources of difference in costs between patients or centres. In a Spanish cost analysis study, also using real-life resources, the cost of MT including predominantly SR devices with consumables and staffing was equivalent to $£ 5,980 .^{23}$ In a Norwegian health technology assessment, the estimated costs of MT including the procedure, diagnostical imaging and transport were estimated to be $£ 5,919$, substantially lower that the estimate in our study of these same components of $£ 8,750 .{ }^{24}$ The higher MT costs in some of the economic analyses above compared with this current study, where a mixture of devices were used, could be related to the predominant use of SR devices for MT.

The study by Vega-Valdes et al, examining the cost of MT in Spain, was similar to our study in using retrospective individual patient data; however, it had a smaller sample size of 105 patients ( 44 patients treated with IVT and 61 with MT). ${ }^{25}$ Their estimated cost of MT was equivalent to $£ 4,528$ per patient, with an average final total cost of $£ 12,070$. The total figure covered the costs of emergency admission, imaging, physician consultation, admission up to 24 hours after intervention, rehabilitation sessions and 90-day outpatient follow-up. In contrast to the current study, they did not include costs across the whole treatment pathway, having excluded transportation and other periprocedural staff costs associated with MT. Overall, this study is more similar to our study than the other procedure-only studies; however, our study was not limited by a small sample and included all components of the care pathway. In summary, past literature appears limited compared with the current study, which comprehensively includes resources and costs across the whole patient pathway including ambulance costs, the intervention, and hospital treatment to discharge. Differences in costs between the present study and previous studies could be due to a range of factors including variations in organisational set-up, geographical location, available resources, type of healthcare infrastructure, national and individual centre device costs (which can vary considerably), and type of models of care (mothership or drip-and ship); some of these are probably further impacted by currency exchange fluctuations.

Our study also provides new insights into variability across centres and between patient groups. The wide variation in the costs of providing MT across the centres arises mainly from a combination of differences in imaging costs (which are calculated differently in different hospitals, especially with regard to capital depreciation etc), relative use of SR (as opposed to other devices), relative use of general anaesthesia and the proportion of MT cases requiring ICU admission. Centres with higher use of general anaesthesia and ICU admission, in particular, had higher costs. This variation could also be partly explained by available resources and the way services are organised; patients may go straight to the stroke unit, or the radiology department bypassing the emergency department; repeat scans may occur for all drip-andship patients or just for few selected cases; all facilities may be on one neuroscience site as opposed to two sites and immediate postMT imaging and 24-hour scans may be performed as opposed to only 24-hour post-MT scans. In addition, costs are not consistent between regions. In the London region, unit costs are uplifted by $13.9 \%$, reflecting the much higher costs of healthcare inputs in the London conurbation than in most of the rest of the UK. ${ }^{26}$ Therefore, organisational and cost variations can significantly affect the total cost of MT at each centre.

After the first 24 hours of care, the variation in costs arises from ICU admissions and subsequent length of stay, particularly for the drip-and-ship patients depending on whether there is a rapid repatriation policy for transfer back to referring primary hospital. Some of these variations could be reviewed in order to make the intervention more cost effective.

The main differences between the mothership and drip-and-ship service paradigm were mainly in costs associated with additional EHA and imaging costs, owing to repeat EHA and computed tomography at the MT centre. The significant difference in costs of transportation in the drip-and-ship group was due to inter-hospital transportation and repatriation costs. There were insufficient numbers to compare mothership and drip-and-ship separately for each of the five centres. The only significant difference between the direct transfer before repatriation and the drip-and-ship 
models of care was primarily driven by the additional costs of repeat imaging.

The mothership and drip-and-ship are the commonly used models of care in most regions in the UK, particularly as identified in this study. The direct transfer model is not widely used but has become common in the London region since the reorganisation of stroke services there in 2010. The direct transfer model has been shown to reduce disability from stroke but, due to the small number of patients receiving that model of care in this study, further evidence on its costs is required particularly in regions outside London. ${ }^{27,28}$

\section{Strengths and limitations}

There were a number of strengths to our approach. Firstly, a bottom-up micro-costing approach was utilised. This allowed for the identification and quantification of resource use from pre-hospital transportation, arrival to hospital, imaging, intervention and hospital care until discharge from neuroscience centre as well as the costs component per individual patient, yielding better insight into drivers of costs, including within some patient subgroups. Additionally, the data were collected from patients treated in routine clinical practice which represented real-world data. Lastly, the fact that the data emanates from multiple centres in different regions, allows considerable potential for generalisation across wider healthcare economies where, as in the current study, differing referral pathways pertain (eg as regards proportionate use of mothership and drip-and-ship). Differing levels of brain imaging, general anaesthesia and of SR use may all apply between centres, regions and countries.

We also recognise some limitations to this study. Firstly, we were unable to collect further resource use or long-term outcome data after discharge from MT centres, particularly on patients repatriated back to their primary hospitals, or on those transferred for rehabilitation into the community. Rehabilitation costs and costs after repatriation up to discharge from primary hospitals are therefore not included and were outside the scope of the current study.

Secondly, the patients and centres included may not be fully representative of all MT patients across the UK, as less than the $1.4 \%$ of patients from SNAPP were included.

\section{Conclusion}

We have provided detailed estimates of the current costs of providing MT in routine clinical settings from a UK NHS perspective. The main drivers of costs are the devices, use of general anaesthesia and admission to ICU/HDU. Our results should be valuable for all healthcare commissioners/funders and decision makers exploring the true cost of providing MT in a clinical setting. This will be particularly relevant in countries, such as the UK, where, for multiple reasons including the historical geographical location of centres (not optimum for MT provision) and shortfalls in required infrastructure and workforce manpower, there are limited numbers of thrombectomy centres. More generally, these findings should be of value for strategic planning/commissioning of stroke services, for future economic evaluations of interventions, and to inform future healthcare policy recommendations.

\section{Supplementary material}

Additional supplementary material may be found in the online version of this article at www.rcpjournals.org/clinmedicine: $\mathrm{S} 1$ - Unit costs and references.

S2 - Costs breakdowns, comparisons and results.

\section{Acknowledgements}

We would like to express our thanks to all participating neuroscience centres and staff.

\section{Conflicts of interest}

Alastair Gray is partly funded by the NIHR Oxford Biomedical Research Centre. Phil White has undertaken consultancy work for Stryker, Codman and MicroVention who manufacture stroke thrombectomy devices. Gary Ford has received personal remuneration for educational and advisory work from Amgen, Daiichi Sankyo, Medtronic, Pfizer and Stryker; and his institution has received educational grants from Medtronic and Pfizer for stroke related activities. Martin James has received honoraria and support for educational activities from Boehringer Ingelheim and Medtronic. Joyce Balami has received a travel grant from Kellogg College, University of Oxford. Alastair Buchan is partly funded by the NIHR Oxford Biomedical Research Centre and is senior medical science advisor and co-founder of Brainomix, a company that develops electronic ASPECTS (e-ASPECTS). Robert Simister is partly funded by the NIHR University College London Hospitals Biomedical Research Centre.

Christine Roffe has received personal remuneration for educational and advisory work from Allergan and Daiichi Sankyo and her institution has received educational grants from Medtronic, Brainomix, Firstkind Medical and Johnson \& Johnson for stroke-related activities. She was the national coordinator for the ECASS-4 study and is the national lead for SITS International, both of which are partially funded by Boehringer Ingelheim.

\section{Funding}

This report is independent research supported by the NIHR Applied Research Collaboration South West Peninsula (PenARC). The views expressed in this publication are those of the authors and not necessarily those of the NIHR or the Department of Health and Social Care.

\section{References}

$1 \mathrm{XuXM}$, Vestesson E, Paley L et al. The economic burden of stroke care in England, Wales and Northern Ireland: Using a national stroke register to estimate and report patient-level health economic outcomes in stroke. Eur Stroke J 2018;3:82-91.

2 Goyal M, Menon BK, van Zwam WH et al. Endovascular thrombectomy after large-vessel ischaemic stroke: a meta-analysis of individual patient data from five randomised trials. Lancet 2016;387:1723-31.

3 Flynn D, Francis R, Halvorsrud $K$ et al. Intra-arterial mechanical thrombectomy stent retrievers and aspiration devices in the treatment of acute ischaemic stroke: A systematic review and metaanalysis with trial sequential analysis. Eur Stroke J 2017;2:308-18.

4 Boudour S, Barral M, Gory B et al. A systematic review of economic evaluations on stent-retriever thrombectomy for acute ischemic stroke. J Neurol 2018:265:1511-20. 
5 Sevick LK, Ghali S, Hill MD et al. Systematic review of the cost and cost-effectiveness of rapid endovascular therapy for acute ischemic stroke. Stroke 2017:48:2519-26.

6 Bracard S, Ducrocq X, Mas JL et al. Mechanical thrombectomy after intravenous alteplase versus alteplase alone after stroke (THRACE): a randomised controlled trial. Lancet Neurol 2016;15: 1138-47.

7 NHS England. Clinical Commissioning Policy. Mechanical thrombectomy for acute ischaemic stroke (all ages) 2018: NHS England Reference: 170033P. NHS, 2018.

8 National Institute for Health and Care Excellence. Mechanical thrombectomy devices for acute ischaemic stroke: Medtech innovation briefing [MIB153]. NICE, 2018.

9 Ford GA, James M, White P (eds). Mechanical thrombectomy for acute ischaemic stroke: an implementation guide for the UK. Oxford Academic Health Science Network, 2019. www.oxfordahsn. org/wp-content/uploads/2019/07/Mechanical-Thrombectomy-forIschaemic-Stroke-August-2019.pdf

10 McMeekin P, White P, James MA et al. Estimating the number of UK stroke patients eligible for endovascular thrombectomy. Eur Stroke J 2017;2:319-26.

11 Gold M, Siegel S, Russell L, Weinstein M (eds). Cost-effectiveness in health and medicine. New York: Oxford University Press, 1996.

12 Drummond M, Sculpher M, Claxton KP, Stoddart G, Torrance G. Methods for the economic evaluation of health care programmes, 4th edn. Oxford: Oxford University Press, 2015.

13 NHS Improvement. Reference costs 2017/18: highlights, analysis and introduction to the data. NHS Improvement, 2018. https:// improvement.nhs.uk/resources/reference-costs

14 National Statistics. GDP deflators at market prices, and money: GDP June 2019 (Quarterly National Accounts). HM Treasury, 2019. www.gov.uk/government/statistics/gdp-deflators-at-market-pricesand-money-gdp-june-2019-quarterly-national-accounts

15 Curtis A, Burns A. Unit cost of health and social care. Canterbury: Personal Social Services Research Unit, 2017.

16 BMJ Group, Royal Pharmaceutical Society. BNF. London: BM] Group, Royal Pharmaceutical Society, 2019. https://bnf.nice.org.uk

17 The World Bank. DataBank. The World Bank. http://databank.worldbank.org/data/reports.aspx? source $=2 \&$ series $=$ PA.NUS.PPP\#

18 Health and Social Care Board. Finance Department internal communication. Belfast: Health and Social Care Board, 2019.

19 NHS England, NHS Improvement. 2019/20 National tariff payment system. NHS, 2019 https://improvement.nhs.uk/resources/ national-tariff/\#h2-201920-national-tariff-payment-system
20 Ganesalingam J, Pizzo E, Morris S et al. Cost-utility analysis of mechanical thrombectomy using stent retrievers in acute ischemic stroke. Stroke 2015;46:2591-8.

21 Lobotesis K, Veltkamp R, Carpenter IH et al. Cost-effectiveness of stent-retriever thrombectomy in combination with IV t-PA compared with IV t-PA alone for acute ischemic stroke in the UK. J Med Econ 2016;19:785-94.

22 Steen Carlsson K, Andsberg G, Petersson J, Norrving B. Long-term cost-effectiveness of thrombectomy for acute ischaemic stroke in real life: An analysis based on data from the Swedish Stroke Register (Riksstroke). Int J Stroke 2017;12:802-14.

23 de Andres-Nogales F, Alvarez M, de Miquel MA et al. Costeffectiveness of mechanical thrombectomy using stent retriever after intravenous tissue plasminogen activator compared with intravenous tissue plasminogen activator alone in the treatment of acute ischaemic stroke due to large vessel occlusion in Spain. Eur Stroke ] 2017;2:272-84.

24 Frønsdal KB, Skår A, Stoinska-Schneider A, Ormstad SS, Fure B. [Mechanical thrombectomy for acute ischemic stroke]. Oslo: Norwegian Institute of Public Health, 2016. www.fhi.no/en/ publ/2016/mekanisk-trombektomi-ved-akutt-hjerneinfarkt

25 Vega-Valdes P, Murias-Quintana E, Morales-Santos E et al. [Analysis of the direct costs associated with mechanical thrombectomy and intravenous fibrinolysis in the Hospital Universitario Central de Asturias]. Rev Neurol 2018;66:7-14.

26 Monitor, NHS England. A guide to the market forces factor. Monitor, 2013. https://assets.publishing.service.gov.uk/ government/uploads/system/uploads/attachment_data/ file/300859/A_guide_to_the_Market_Forces_Factor.pdf

27 Bray BD, Ayis S, Campbell J et al. Associations between the organisation of stroke services, process of care, and mortality in England: prospective cohort study. BMJ 2013;346:f2827.

28 Morris S, Hunter RM, Ramsay AI et al. Impact of centralising acute stroke services in English metropolitan areas on mortality and length of hospital stay: difference-in-differences analysis. BMJ 2014;349:94757.

Address for correspondence: Dr Joyce S Balami, Centre for Evidence-Based Medicine, University of Oxford, 1 Wellington Square, Oxford, Oxfordshire OX1 2JA, UK. Email: joyce.balami@kellogg.ox.ac.uk 